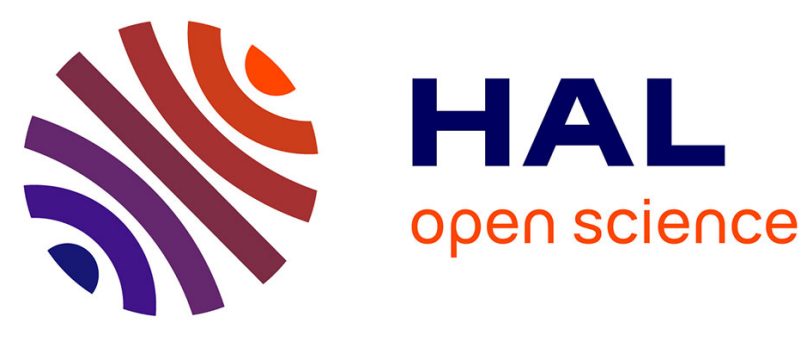

\title{
61 channels coherent beam combining femtosecond digital laser
}

Jean-Christophe Chanteloup, Severine Bellanger, Louis Daniault, Ihsan Fsaifes, Matthieu Veinhard, Jérôme Bourderionnet, Christian Larat, Eric Lallier, Arnaud Brignon

\section{To cite this version:}

Jean-Christophe Chanteloup, Severine Bellanger, Louis Daniault, Ihsan Fsaifes, Matthieu Veinhard, et al.. 61 channels coherent beam combining femtosecond digital laser. SPIE Photonics West, SPIE, Mar 2021, san francisco, United States. 10.1117/12.2576606 . hal-03368944

\section{HAL Id: hal-03368944 https://hal.science/hal-03368944}

Submitted on 7 Oct 2021

HAL is a multi-disciplinary open access archive for the deposit and dissemination of scientific research documents, whether they are published or not. The documents may come from teaching and research institutions in France or abroad, or from public or private research centers.
L'archive ouverte pluridisciplinaire $\mathbf{H A L}$, est destinée au dépôt et à la diffusion de documents scientifiques de niveau recherche, publiés ou non, émanant des établissements d'enseignement et de recherche français ou étrangers, des laboratoires publics ou privés. 


\section{1 channels coherent beam combining femtosecond digital laser}

Chanteloup, Jean-Christophe, Bellanger, Séverine, Daniault, Louis, Fsaifes, Ihsan, Veinhard, Matthieu, et al.

Jean-Christophe Chanteloup, Séverine Bellanger, Louis Daniault, Ihsan Fsaifes, Matthieu Veinhard, Jérôme Bourderionnet, Christian Larat, Eric Lallier, Arnaud Brignon, "61 channels coherent beam combining femtosecond digital laser," Proc. SPIE 11665, Fiber Lasers XVIII: Technology and Systems, 116651H (5 March 2021); doi: 10.1117/12.2576606

SPIE. Event: SPIE LASE, 2021, Online Only 


\title{
61 channels Coherent Beam Combining femtosecond Digital laser
}

\author{
Jean-Christophe Chanteloup ${ }^{1}$, Séverine Bellanger ${ }^{1}$, Louis Daniault ${ }^{1}$, Ihsan Fsaifes ${ }^{1}$, Matthieu \\ Veinhard ${ }^{1}$, Jérôme Bourderionnet ${ }^{2}$, Christian Larat $^{2}$, Eric Lallier ${ }^{2}$, Arnaud Brignon ${ }^{2}$ \\ ${ }^{1}$ LULI, CNRS, Ecole Polytechnique, CEA, Sorbonne Université, Institut Polytechnique de Paris, \\ 91128 Palaiseau, France \\ ${ }^{2}$ Thales Research \& Technology, 1 avenue Augustin Fresnel, 91767 Palaiseau Cedex, France
}

\begin{abstract}
Tiled-aperture Coherent Beam Combination architecture opens the way to digital laser operating in high peak and average power regimes.
\end{abstract}

\section{INTRODUCTION}

Tailoring light distribution to match specific experimental or industrial needs is a quest as ancient as optical engineering exists. However, it is not until its invention of the laser in 1960 [1] that humankind could start taking advantage of coherent light towards this end. Although the first fiber laser was reported soon after [2], it did not appear on the market until the late 80s, simultaneously with the Chirp Pulse Amplification (CPA) technique [3] allowing the amplification of ultrashort pulses by stretching the power distribution over time. High (GW to PW) laser peak powers CPA based laser allowed many scientific breakthroughs over the recent decades, but the path towards commercial and/or societal applications has been limited for years by the lack of average power delivered by state of the art laser devices (a few $\mathrm{kHz}$ for Ti:sapphire based amplifying systems for example). Considering for instance acceleration of particles, one shall observe that, if first demonstrations [4] relied on low repetition rate $(1 \mathrm{~Hz})$ PW laser system like Bella at LNBL [5], foreseen particle accelerators would require to operate the laser at repetition rates orders of magnitude higher to reach the 100's of $\mathrm{kW}$ average power requested (see table 25 of [6]). But getting both high peak and average powers simultaneously becomes now a reality thanks to tremendous progresses in high power ultrafast thin disk lasers [7] but also a more scalable laser architectural approach called Coherent Beam Combining (CBC) [8-10] with the kW average power horizon already exceeded for both technologies.
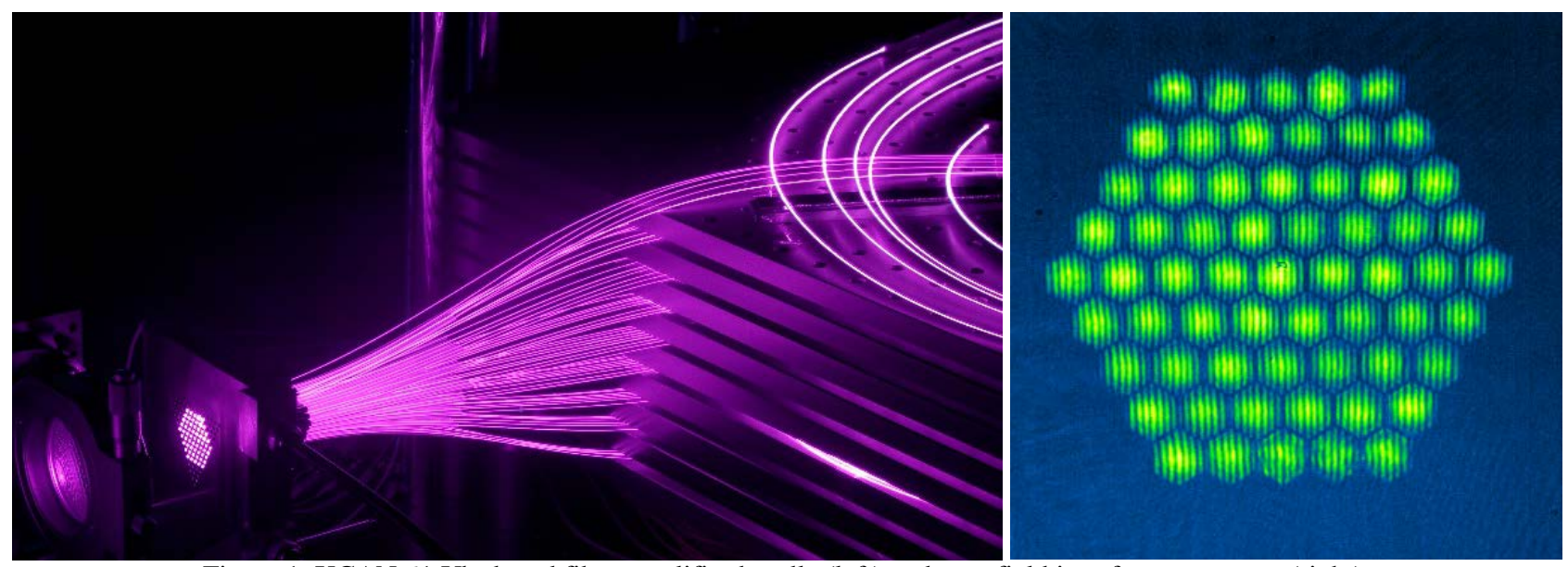

Figure 1. XCAN 61 Yb-doped fiber amplifier bundle (left) and near field interference pattern (right). 
Within this context, Ecole Polytechnique and the Thales company have developed a CBC 61 channels prototype called XCAN [11] relying on Tiled-apertures allowing single step far field combination (figure 1, left). The CBC is indeed simply achieved when focusing the composite large pupil (Figure 1, right) with a single lens. The electric field transverse distribution observed in this lens focal plane indeed results from the coherent addition of all 61 beams accurately phased together. When compared to Filled-aperture [12], Tiled-aperture CBC is inherently limited in efficiency to the power concentrated into the main lobe in the far field but offers an unrivaled agility in terms of far field beam shaping. Indeed, each channel shall be seen as an individual pixel where amplitude, phase and polarization can be addressed independently. Thanks to its highly scalable tiled aperture architecture, this prototype opens the path to a new era of full digital lasers with unmatched potential for light shaping in various power regimes.

Adjusting light distribution to optimize material processes is an important industrial request with very different peculiar shaping requirements [13]. Although we have shown that XCAN architectural approach can generate arbitrary shaped light distribution (see last figure of [14]), we have experimentally focused our demonstration on Orbital Angular Momentum (OAM) laser beams generation. Such beams carry indeed helical phase fronts [15]. The hexagonal tiling distribution of our laser appears therefore perfectly suited for such phase symmetry generation. Moreover, there exists a wide range of applications from optical manipulation (i.e. 2018 Nobel prize in physics granted to A.Ashkin for optical tweezer [16], and shared with D.Strickland \& G.Mourou for above mentioned CPA), quantum optics [17], imaging [18] (like in astronomy [19] or fluids flow characterization [20]), optical communications [21]/cryptography [22]. One shall also consider applications requiring much higher power regime like microbunching instability reduction in free-electron laser setups [23] or propagation of ultraintense laser beams [24] (for channeling lightning strikes or detection of atmospheric pollutant [25-26]).

Traditional methods to generate OAM beams rely generally on laser cavity tuning [27], phase plates [28], axicons [29] and Spatial-Light Modulators (SLMs) [30-31]. The first ones allow high output powers regime operation but lacks tunability, as a different cavity design or optical element must be engineered for each specific transverse beam distribution. The last method offers high tunability [32] but is limited in output powers (peak and average) by the SLM optical damage thresholds. We believe XCAN digital laser is a far more versatile alternative approach, offering both high throughput power and tunability.

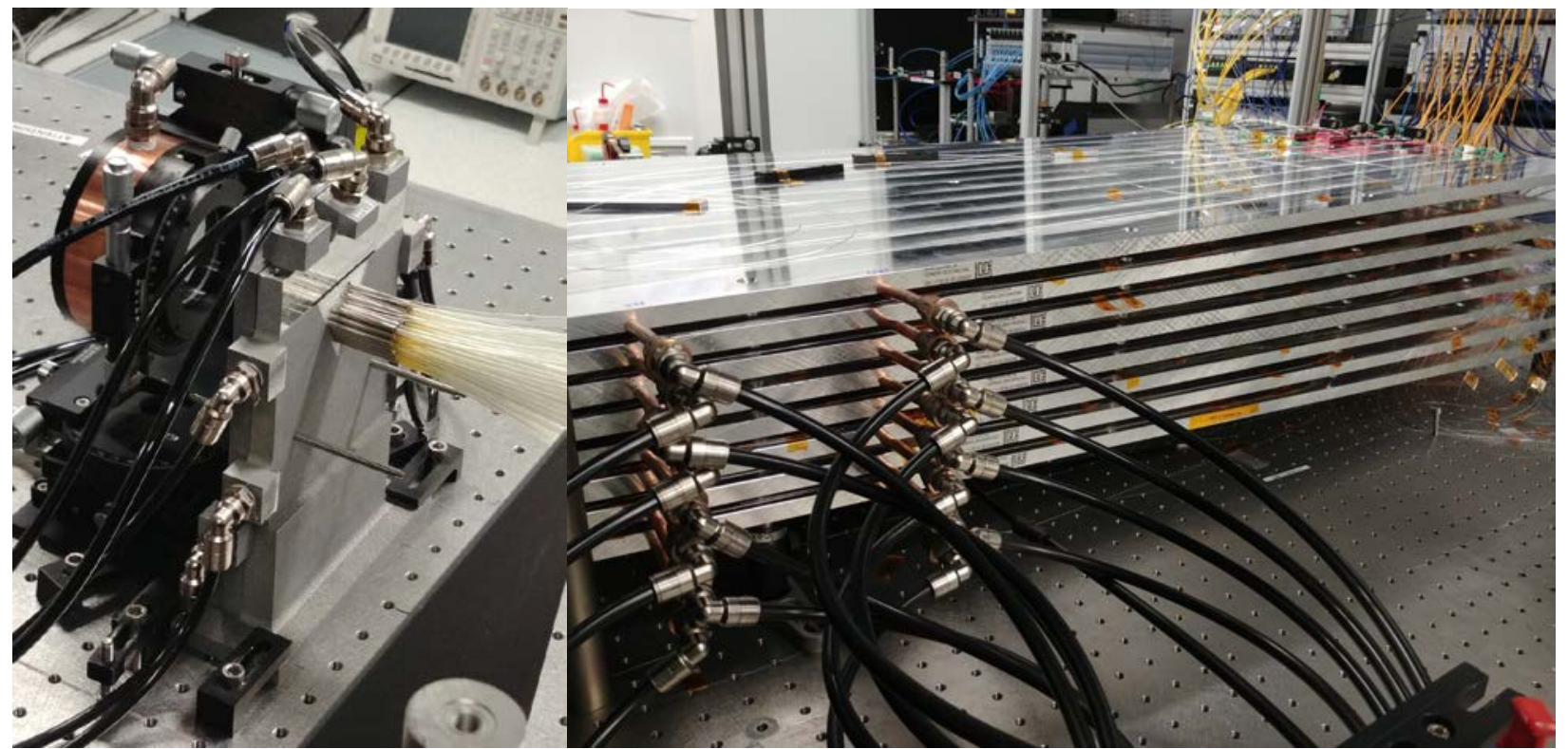

Figure 2. Water-cooled XCAN amplifiers supporting plates (right). Water-cooled 3D printed Aluminium laser head and microlens array (left). 


\section{LASER CHAIN THERMAL MANAGEMENT}

XCAN undergone a complete active thermal cooling upgrade in order to surpass the previously achieved CBC efficiency: $48 \%$ at $10 \mathrm{~W} / 16 \%$ at $1 \mathrm{~kW}$ [11]. All nine amplifiers supporting plates, the laser head and the microlens array mounts are now water-cooled as illustrated on figure 2. It is therefore possible to operate the laser chain in the $\mathrm{kW}$ regime at CBC efficiency level similar to low power as illustrated on figure 3 where the laser was operating during an hour with a stable efficiency above $43 \%$.
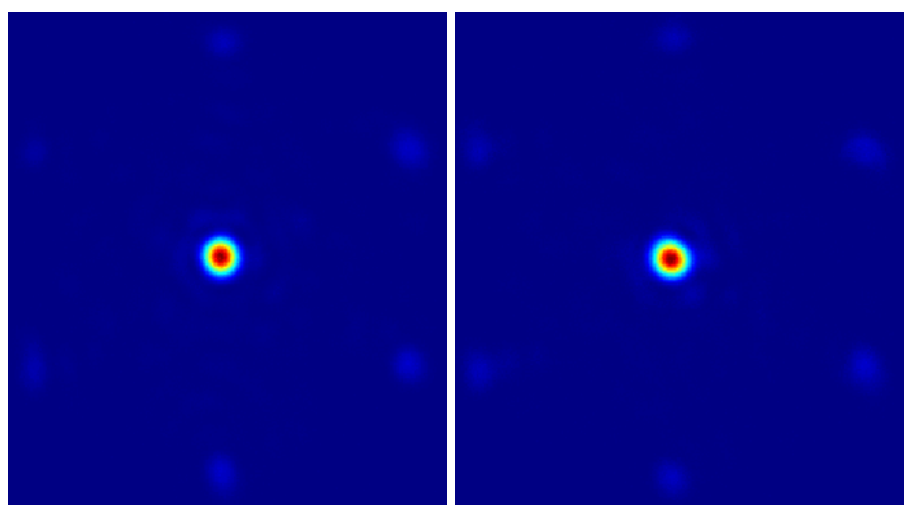

Figure 3. Far field at 671W and 1046W. Respective efficiencies are 49 and 43\%, maintained during an hour operation for the latest.

\section{DIGITAL LASER OPERATIONS}

When applying a helicoidal phase distribution in place of a flat one, Orbital Angular Momentum beams with characteristic donut shape transverse distributions displayed on figure 4 (low power) and 5 (high power) can be generated.
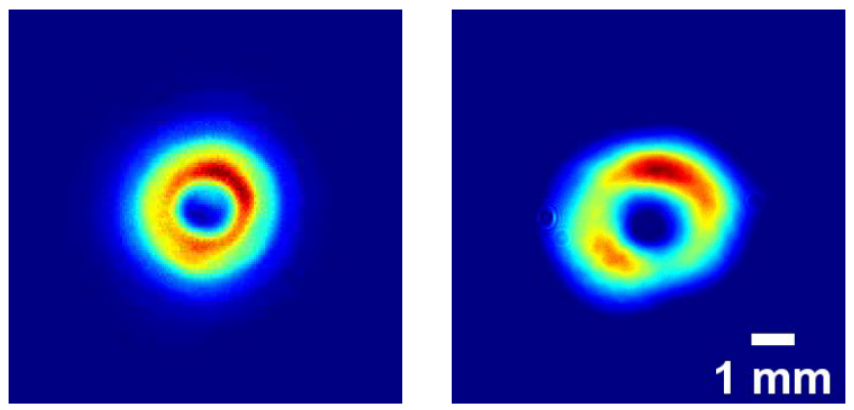

Figure 4. OAM beam profile before (2.5 ns) and after compression (<400 fs). 


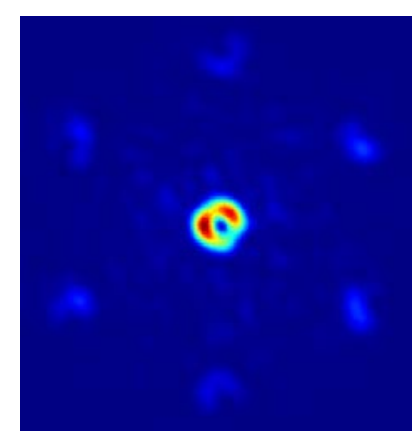

Figure 5. OAM beam profile before at 1043W average power level.

We believe XCAN highly scalable architecture shall be considered as the advent of a new laser architecture paradigm: the digital laser. Such approach carries a high potential for greatly broadening the field of applications of structured light beyond OAM centrosymmetric beams only [20, 23, 33]. It indeed associates high peak (fs regime operation) and average (kW regime) powers thanks to its diode-pumped Yb-doped fiber amplifying chain architecture. Compact and scalable by nature, it has been conceived and implemented to be compatible with up to 10,000 channels paving the way to high-resolution transverse amplitude and phase laser beam control. Finally, these beam properties real time adjustability (currently up to the $\mathrm{kHz}$ regime) are offering an extra degree of freedom for applications (drilling, cutting or soldering of material for instance) requesting dynamic energy or power distribution control.

\section{REFERENCES}

[1] T. Maiman, "Stimulated Optical Radiation in Ruby”, Nature, Vol. 187, pp. $493-494$ (1960).

[2] C. J. Koester and E. Snitzer, “Amplification in a Fiber Laser”, Applied optics, Vol. 3, Issue 10, pp. 1182-1186 (1964).

[3] D. Strickland and G. Mourou, “Compression of amplified chirped optical pulses”, Optics Communications, Vol. 56, No. 3, pp. 219-221 (1985).

[4] W.P. Leemans et al. (2006). "GeV electron beams from a centimetre-scale accelerator,” Nature Physics, Vol. 418, pp. 696-699 (2006).

[5] K. Nakamura et al., "Diagnostics, Control and Performance Parameters for the BELLA High Repetition Rate Petawatt Class Laser,” IEEE Journal of Quantum Electronics, Vol. 53, p. 1200121 (2017).

[6] W.P. Leemans, "White paper of the ICFA-ICUIL joint task force: High power laser technology for accelerators,” ICFA Beam Dyn.Newslett, Vol. 56, pp. 10-88 (2011). (http://icfabd.kek.jp/WhitePaper_final.pdf)

[7] C. Saraceno et al., "The amazing progress of high-power ultrafast thin-disk lasers”, J. Eur. Opt. Soc.-Rapid Publ. 15, 15 (2019).

[8] T.Y. Fan, “Laser beam combining for high-power, high-radiance sources,” IEEE J. Sel. Top. Quantum Electron., Vol. 11, pp. 567-577 (2005).

[9] L. Lombard et al., "Coherent beam combination of narrow-linewidth $1.5 \mu \mathrm{m}$ fiber amplifiers in a long-pulse regime”, Opt. Lett., Vol. 36, pp. 523-525 (2011).

[10] L. Daniault et al., “Coherent beam combining of two femtosecond fiber chirped-pulse amplifiers”, Opt. Lett., Vol. 36, pp. 621-623 (2011).

[11]I. Fsaifes et al., “Coherent beam combining of 61 femtosecond fiber amplifiers”, Optics Express, Vol. 28, No. 14, pp. 20152-20161 (2020).

[12] M. Müller et al., “10.4 kW coherently combined ultrafast fiber laser”, Opt. Lett., Vol. 45, pp. 3083-3086 (2020).

[13]F. O. Olsen et al., “Multibeam fiber laser cutting”, Journal of Laser Applications”, Vol. 21, p. 133 (2009). 
[14] M. Veinhard et al., "Orbital Angular Momentum beams generation from 61 channels Coherent Beam Combining femtosecond Digital Laser”, Optics Letters, Vol. 46, No.1, pp.25-28 (2021)

[15] L. Allen, et al., "Orbital angular momentum of light and the transformation of Laguerre-Gaussian laser modes”, Phys. Rev. A Vol. 45, No. 11, pp. 8185-8189 (1992).

[16] A. Ashkin et al., "Observation of a single-beam gradient force optical trap for dielectric particles”, Opt. Lett., Vol. 11, No 5, pp. 288-290 (1986).

[17] N. M. Litchinitser, “Applied physics. Structured light meets structured matter” Science 337(6098), 1054-1055 (2012).

[18] L. Torner et al., "Digital spiral imaging,” Opt. Express 13(3), 873-881 (2005).

[19] G. A. Swartzlander et al., “Astronomical demonstration of an optical vortex coronagraph”, Opt. Express, Vol. 16, No. 14, pp. 10200-10207 (2008).

[20] A. Ryabtsev et al., "Fluid flow vorticity measurement using laser beams with orbital angular momentum”, Opt. Express, Vol. 24, pp. 11762-11767 (2016).

[21] A. E. Willner et al., "Optical communications usingorbital angular momentum beams”, Adv. Opt. Photonics, Vol. 7, No. 1, p. 66 (2015).

[22] M. Mirhosseini et al., "High-dimensional quantum cryptography with twisted light”, New J.Phys., Vol. 17, No. 3, p. 033033 (2015).

[23] N. Liebster, J. Tang, D. Ratner, W. Liu, S. Vetter, Z. Huang, and S. Carbajo, “Laguerre-Gaussian and beamlet array as second generation laser heater profiles ”Phys. Rev. Accel. Beams, Vol. 21, p. 090701 (2018).

[24] C. Ament et al., "Generation of multiterawatt vortex laser beams”, Applied Optics, Vol. 53, Issue 15, pp. 3355-3360 (2014)

[25] A. Couairon and A. Mysyrowicz, "Femtosecond filamentation in transparent media”, Phys. Rep., Vol. 441, No. 47 (2007).

[26] L. Berge et al., "Ultrashort filaments of light in weakly ionized, optically transparent media”, Rep. Prog. Phys., Vol. 70, p. 1633 (2007).

[27] A. Mock et al., "Tunable Orbital Angular Momentum Radiation from Angular-Momentum-Biased Microcavities," Phys. Rev. Lett., Vol. 121, p. 103901 (2018).

[28] C. Dorrer, "High-damage-threshold beam shaping using binary phase plates", Opt. Lett., Vol. 34, pp. 2330-2332 (2009).

[29] N. Ochiai et al., “Axicon-based beam shaping for low-loss nonlinear microscopic optics”, J. Opt. Soc. Am. B, Vol. 36, pp. 1342-1347 (2019).

[30] L. Zhu et al., “Arbitrary manipulation of spatial amplitude and phase using phase-only spatial light modulators”, Sci. Rep., Vol. 4, p. 7441 (2015).

[31] R. Lemons et al., "Programmable Control of Femtosecond Structured Light”, Conference on lasers and electrooptics, 2019, pp. SW4E.6

[32] S. Ngcobo et al., “A digital laser for on-demand laser modes”, Nat. Commun. 4:2289 (2013).

[33] G. Tkachenko, E. Brasselet, "Helicity-dependent three-dimensional optical trapping of chiral microparticles." Nat Commun., Vol. 5, p. 4491 (2014). 